\title{
Analytical Solution to the Large Deformation of Cantilever Beams with Two Angled Point Forces
}

\author{
Jennifer Lew \\ Palos Verdes Peninsula High School \\ Rancho Palos Verdes, CA, USA \\ jenniferlew2004@gmail.com \\ Project Advisor: Derek Fong, PE \\ Senior Utilities Engineer, Supervisor \\ California Public Utilities Commission \\ Los Angeles, CA, USA \\ derek.fong@cpuc.ca.gov
}

\begin{abstract}
The analytical solution for the large deformation of a cantilever beam under a point load, typically applied to the tip of the cantilever and perpendicularly to its axis, has been widely studied and published. However, the more complex case of two angled point loads applied to the cantilever has not been published. The current research delved into the following scenario: an upright cantilever, e.g. a pole, has point loads applied at two locations on the cantilever, where each point load is angled, i.e. the point load has both a horizontal component (which may result from wind loading) and downward vertical component of force (such as from weights). The aim of the research is to develop a methodology for finding, at the two locations where the point loads are applied, the angle of deflection, the horizontal deflection, and the vertically deflected height. Ultimately, the research yielded a methodology based on the Complete and Incomplete Elliptic Integrals of the First Kind and Second Kind. The analytical solution developed in this research - specifically the method for calculating the angles of deflections was compared against Finite Element Analysis and was found to produce nearly identical results. We conclude that the methodology shown can be extended to any number of point loads and will be a contribution to the field of non-linear mechanics.
\end{abstract}




\section{Contents}

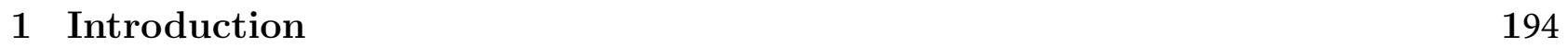

2 Large Deformation using One Prismatic Element 194

$2.1 \quad$ Angle of Deflection at the Element Tip . . . . . . . . . . . . . . . . . . . . . 195

2.2 Horizontal Deflection at the Pole Tip . . . . . . . . . . . . . . . . . . . . . . 199

2.3 Vertically Deflected Height at the Pole Tip . . . . . . . . . . . . . . . . . . . 201

3 Large Deformation using Two Prismatic Elements 202

3.1 Angles of Deflection at the Element Tips . . . . . . . . . . . . . . . . . 202

3.2 Solving for $\delta_{x 1}$, the Horizontal Deflection of Segment 1 . . . . . . . . . . . . 206

3.3 Solving for $a$, the Vertically Deflected Height of Segment 1 . . . . . . . . . . 207

3.4 Solving for $\delta_{x 2}$, the Horizontal Deflection of Segment 2 . . . . . . . . . . . . 208

3.5 Solving for $b$, the Vertically Deflected Height of Segment 2 . . . . . . . . . . 215

4 Validation: Comparing the Analytical Solution to FEA 209

\begin{tabular}{|lll}
5 & Conclusion & 211
\end{tabular}

\begin{tabular}{ll}
\hline References & 212
\end{tabular} 


\section{Introduction}

The analytical solution to the large deformation of a cantilever beam under a tip load, i.e. a point load or force applied to the tip of the cantilever, has been studied thoroughly, as shown in [1 - 7]. Another popular topic of study is the large deformation of a cantilever under a tip load and a distributed load, as shown in [8] - [12]. Indeed, even a master's thesis has been written on the subject of a cantilever beam under a single tip load, as in [13]. The current research examines a novel and more complex topic: the large deformation of a cantilever due to two angled point loads. Specifically, the research proposes this problem: an upright cantilever, e.g. a pole, has two angled point loads, meaning each point load has both a horizontal and vertical component of force. See Figure 3 , right image. The motivation for the study is that solving the problem of two angled point loads (where the angles do not have to be the same) provides a roadmap for solving the problem of any number of angled point loads along the cantilever. The result of this work is that the analytical solution for the proposed problem has been found: the work provides methodology that can be used to calculate the cantilever horizontal deflection, vertical deflection, and tip angle at each angled point load. This work is of interest to persons involved in the design and construction of utility poles, transmission towers, cell towers, antennas, and any other pole-like structure that has multiple horizontal loads (such as wind blowing on exposed surfaces) and vertical loads (such as the weight of the structure or its attachments).

Here, we will briefly discuss two important developmental steps in non-linear mechanics to see how the current work is novel. Before we do so, we will observe the following two definitions: (1) non-angled: this means that, prior to cantilever deflection, the force is exactly perpendicular to the length of the cantilever; (2) angled: this means that, prior to cantilever deflection, the force is at some arbitrary angle to the length of the cantilever. One of the earliest works exploring the deformation of a cantilever is [14] from Bisshopp and Drucker. In this paper, the authors describe a solution for the deflection of a cantilever under one non-angled point load at the tip of the cantilever. Another well-known work is [15] from Frisch-Fay. The author describes a solution for the deflection of a cantilever under two or more angled point loads as long as all of the loads are parallel to each other. By using parallel loads, the author was able to provide a simplified approach utilizing the principle of elastic similarity. In the current work, we provide essentially the general solution for solving the deflection of a cantilever under an arbitrary loading condition. This general solution is not provided in [14] or [15. The solution in the current work can be extended to any number of loads, with each load able to have an arbitrary and different angle.

\section{Large Deformation using One Prismatic Element}

We first consider a pole with a tip load, where the tip load is comprised of both a horizontal force $P_{T}$ and a vertical force $P_{V}$. The pole is treated as a single prismatic element of length $L$. This scenario is shown in Figure 1 below. We will determine, at the pole tip, the angle of deflection $\phi_{o}$, the horizontal deflection $\delta_{x}$, and the vertically deflected height $L-\delta_{y}$. 


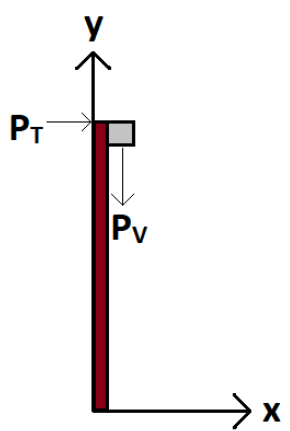

(a)

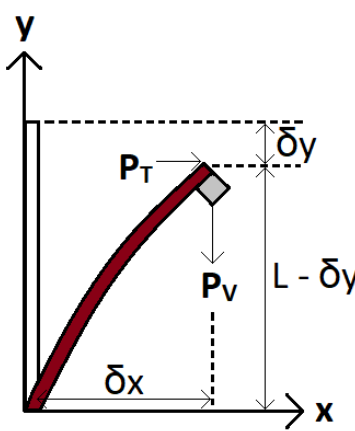

(b)

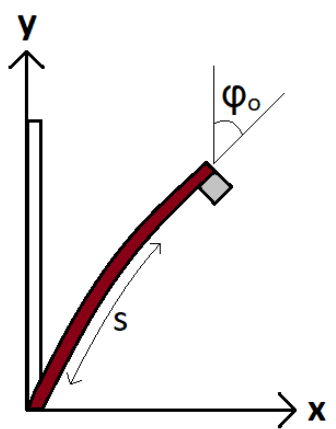

(c)

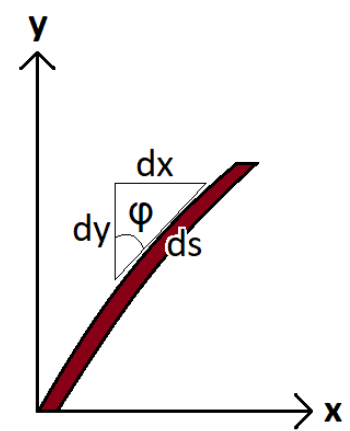

(d)

Figure 1: (a) Prismatic pole with a horizontal (or transverse) load and a vertical load, prior to deflection. (b) The pole after deflection. (c) The arc length $s$ and the angle of deflection $\phi$. Note that the angle of deflection at the pole tip is denoted as $\phi_{o}$. (d) Here, we show a small, approximately straight-line segment.

\subsection{Angle of Deflection at the Element Tip}

As a first step toward determining a pole's vertical and horizontal deflection, let us calculate $\phi_{o}$. We define $E$ as the pole's elasticity and $I$ as the pole's moment of inertia (or second moment of area). Bernoulli-Euler beam theory gives the following relationship between a beam's curvature, $d \phi / d s$, and its bending moment, $M$.

$$
M=E I \cdot \frac{d \phi}{d s}
$$

To work with differentials, let us differentiate both sides of (1) with respect to $s$, as follows:

$$
\frac{d M}{d s}=E I \cdot \frac{d^{2} \phi}{d s^{2}}
$$

The bending moment at any location $(\mathrm{x}, \mathrm{y})$ along the pole is:

$$
M(s)=P_{T} \cdot\left(L-\delta_{y}-y\right)+P_{V} \cdot\left(\delta_{x}-x\right)
$$

Differentiating both sides of (3) gives:

$$
\frac{d M}{d s}=P_{T} \cdot\left(\frac{d}{d s} L-\frac{d}{d s} \delta_{y}-\frac{d y}{d s}\right)+P_{V} \cdot\left(\frac{d}{d s} \delta_{x}-\frac{d x}{d s}\right)
$$

By examining Figure 1(d) we can obtain the following two relationships:

$$
\begin{aligned}
& \frac{d x}{d s}=\sin \phi \\
& \frac{d y}{d s}=\cos \phi
\end{aligned}
$$

Substituting (5) and (6) into (4), we obtain:

$$
\frac{d M}{d s}=-P_{T} \cdot \cos \phi-P_{V} \cdot \sin \phi
$$


Substituting (7) into (2), we obtain:

$$
E I \cdot \frac{d^{2} \phi}{d s^{2}}+P_{T} \cdot \cos \phi+P_{V} \cdot \sin \phi=0
$$

Multiply both sides of (8) by $d \phi / d s$ to obtain:

$$
E I \cdot \frac{d^{2} \phi}{d s^{2}} \cdot \frac{d \phi}{d s}+P_{T} \cdot \cos \phi \cdot \frac{d \phi}{d s}+P_{V} \cdot \sin \phi \cdot \frac{d \phi}{d s}=0
$$

We can rewrite this as follows:

$$
\frac{d}{d s}\left[\frac{1}{2} \cdot E I \cdot\left(\frac{d \phi}{d s}\right)^{2}+P_{T} \cdot \sin \phi-P_{V} \cdot \cos \phi\right]=0
$$

From which we obtain:

$$
\frac{1}{2} \cdot E I \cdot\left(\frac{d \phi}{d s}\right)^{2}+P_{T} \cdot \sin \phi-P_{V} \cdot \cos \phi=C
$$

In order to determine the constant $C$, we use the condition that when $(\phi, \mathrm{s})=\left(\phi_{o}, \mathrm{~L}\right)$, it follows that $d \phi / d s=0$. This indicates that at the tip of the beam, the curvature is 0, i.e. the radius of curvature is infinity because if we were to extend the tip of the beam, that extension would continue in a straight line. Applying our condition, we can find our constant as:

$$
C=P_{T} \cdot \sin \phi_{o}-P_{V} \cdot \cos \phi_{o}
$$

We substitute (12) into (11) and solve for $d s$ :

$$
d s=\sqrt{\frac{E I}{2}} \cdot \frac{d \phi}{\sqrt{P_{T} \cdot \sin \phi_{o}-P_{T} \cdot \sin \phi-P_{V} \cdot \cos \phi_{o}+P_{V} \cdot \cos \phi}}
$$

By performing a summation of sinusoids in the denominator of the second factor, 13 can be simplified as follows:

$$
d s=\sqrt{\frac{E I}{2}} \cdot \frac{d \phi}{\sqrt{P_{R} \cdot \sin \left(\phi_{o}+\beta\right)-P_{R} \cdot \sin (\phi+\beta)}}
$$

where

$$
\begin{aligned}
P_{R} & =\sqrt{P_{T}^{2}+P_{V}^{2}} \\
\beta & =\arcsin \left(\frac{-P_{V}}{\sqrt{P_{T}^{2}+P_{V}^{2}}}\right)
\end{aligned}
$$

We can integrate both sides of (14) along the entire length of the pole, starting from the base where $\phi=0$ to the pole tip, where $\phi=\phi_{o}$.

$$
L=\sqrt{\frac{E I}{2}} \cdot \int_{0}^{\phi_{o}} \frac{d \phi}{\sqrt{P_{R} \cdot \sin \left(\phi_{o}+\beta\right)-P_{R} \cdot \sin (\phi+\beta)}}
$$


If we further define a value $\alpha$ as:

$$
\alpha=\frac{P_{R} \cdot L^{2}}{E I}
$$

then $(17)$ can be rewritten as:

$$
\sqrt{\alpha}=\int_{0}^{\phi_{o}} \frac{1}{\sqrt{2}} \cdot \frac{d \phi}{\sqrt{\sin \left(\phi_{o}+\beta\right)-\sin (\phi+\beta)}}
$$

As it stands, (19) is not integrable and has no known function approximation. However, it can be simplified into an elliptic integral which does have a function approximation. The following equations will show, step by step, how to perform the needed manipulations. First, in $(19)$, we multiply the numerator and denominator of the integrand by a cosine value:

$$
\sqrt{\alpha}=\int_{0}^{\phi_{o}} \frac{1}{\sqrt{2}} \cdot \frac{\cos (\phi+\beta) \cdot d \phi}{\sqrt{1-\sin ^{2}(\phi+\beta)} \cdot \sqrt{\sin \left(\phi_{o}+\beta\right)-\sin (\phi+\beta)}}
$$

The first term in the denominator of $(20)$ can be factorized, and then the integrand rearranged, as follows:

$$
\begin{aligned}
\sqrt{\alpha}= & \int_{0}^{\phi_{o}} \frac{1}{\sqrt{2}} \cdot \frac{1}{\sqrt{1+\sin (\phi+\beta)} \cdot \sqrt{\sin \left(\phi_{o}+\beta\right)-\sin (\phi+\beta)}} \\
& \cdot \frac{\cos (\phi+\beta) \cdot d \phi}{\sqrt{1-\sin (\phi+\beta)}}
\end{aligned}
$$

In (21), we multiply both the numerator and denominator of the integrand by the same sine value:

$$
\begin{aligned}
\sqrt{\alpha}= & \int_{0}^{\phi_{o}} \frac{1}{\sqrt{2}} \cdot \frac{1+\sin \left(\phi_{o}+\beta\right)}{\sqrt{1+\sin (\phi+\beta)} \cdot \sqrt{\sin \left(\phi_{o}+\beta\right)-\sin (\phi+\beta)}} \\
& \cdot \frac{\cos (\phi+\beta) \cdot d \phi}{\left[1+\sin \left(\phi_{o}+\beta\right)\right] \cdot \sqrt{1-\sin (\phi+\beta)}}
\end{aligned}
$$

Now here is where we do a substitution. Let the following be true:

$$
\sin \psi=\sqrt{\frac{1+\sin (\phi+\beta)}{1+\sin \left(\phi_{o}+\beta\right)}}
$$

By using a sine identity, (23) also yields:

$$
\cos \psi=\sqrt{\frac{\sin \left(\phi_{o}+\beta\right)-\sin (\phi+\beta)}{1+\sin \left(\phi_{o}+\beta\right)}}
$$

Using both (23) and (24), we can also write:

$$
\sin 2 \psi=2 \cdot \frac{\sqrt{1+\sin (\phi+\beta)} \cdot \sqrt{\sin \left(\phi_{o}+\beta\right)-\sin (\phi+\beta)}}{1+\sin \left(\phi_{o}+\beta\right)}
$$


Squaring both sides of 23 gives:

$$
\sin ^{2} \psi=\frac{1+\sin (\phi+\beta)}{1+\sin \left(\phi_{o}+\beta\right)}
$$

Taking the derivative of both sides of (26) gives:

$$
\sin 2 \psi d \psi=\frac{\cos (\phi+\beta)}{1+\sin \left(\phi_{o}+\beta\right)} \cdot d \phi
$$

Substituting (25) and (27) into (22) and simplifying, we get:

$$
\begin{aligned}
\sqrt{\alpha} & =\int_{0}^{\phi_{o}} \frac{\sqrt{2} \cdot d \psi}{\sqrt{1-\sin (\phi+\beta)}} \\
& =\int_{0}^{\phi_{o}} \frac{d \psi}{\sqrt{1-\frac{1+\sin (\phi+\beta)}{2}}}
\end{aligned}
$$

Note that we have not yet changed the limits of integration. We will wait until we make a second substitution. At this point, we invent a value $k$ such that the following be true:

$$
k^{2} \cdot \sin ^{2} \psi=\frac{1+\sin (\phi+\beta)}{2}
$$

Substitute 29 into (28) to obtain:

$$
\sqrt{\alpha}=\int_{\phi=0}^{\phi=\phi_{o}} \frac{d \psi}{\sqrt{1-k^{2} \cdot \sin ^{2}}}
$$

Now we can change the limits of integration. Substituting $\phi=\phi_{o}$ into (26) and solving for $\psi$, the upper limit of integration becomes:

$$
\begin{aligned}
\psi\left(\phi=\phi_{o}\right) & =\arcsin \sqrt{\frac{1+\sin \left(\phi_{o}+\beta\right)}{1+\sin \left(\phi_{o}+\beta\right)}} \\
& =\frac{\pi}{2}
\end{aligned}
$$

Substituting $\phi=0$ into 29 and solving for $\psi$, the lower limit of integration becomes:

$$
\psi(\phi=0)=\psi_{o}=\arcsin \left(\frac{1}{\sqrt{2} \cdot k} \cdot \sqrt{1+\sin \beta}\right)
$$

We can rewrite 30 as:

$$
\sqrt{\alpha}=\int_{\psi_{o}}^{\frac{\pi}{2}} \frac{d \psi}{\sqrt{1-k^{2} \cdot \sin ^{2}}}
$$

Here let us make a quick note about $k$ - it is some constant whose value is unknown (we will explain how to deal with this later). However, we are able to determine a range of possible values for $k$. By substituting (26) into (29) and solving for $k$, we find:

$$
k=\frac{1}{\sqrt{2}} \cdot \sqrt{1+\sin \left(\phi_{o}+\beta\right)}
$$


Since we have $0 \leq \sin \left(\phi_{o}+\beta\right) \leq 1$ we deduce that $\sqrt{2} / 2 \leq k \leq 1$. Now, we observe that (33) can be rewritten as follows:

$$
\sqrt{\alpha}=\int_{0}^{\frac{\pi}{2}} \frac{d \psi}{\sqrt{1-k^{2} \cdot \sin ^{2}}}-\int_{0}^{\psi_{o}} \frac{d \psi}{\sqrt{1-k^{2} \cdot \sin ^{2}}}
$$

We note that in (35) the first term is the Complete Elliptic Integral of the First Kind, and the second term is the Incomplete Elliptic Integral of the First Kind. With this in mind, we can rewrite (35) as:

$$
\sqrt{\alpha}=F_{n}\left(\frac{\pi}{2}, k, 0\right)-F_{n}\left(\psi_{o}, k, 0\right)
$$

where:

$$
\begin{aligned}
F_{n}\left(\frac{\pi}{2}, k, 0\right)= & \frac{\pi}{2(2 n+1)} \cdot\left[1+2 \cdot \sum_{m=1}^{n}\left(1-\left[k \cdot \sin \left(\frac{m \pi}{2 n+1}\right)\right]^{2}\right)^{-1 / 2}\right] \\
F_{n}\left(\psi_{o}, k, 0\right)= & \frac{1}{2 n+1} \cdot\left[\psi_{o}+2 \cdot \sum_{m=1}^{n}\left(1-\left[k \cdot \sin \left(\frac{m \pi}{2 n+1}\right)\right]^{2}\right)^{-1 / 2}\right. \\
& \left.\cdot \arctan \left\{\tan \psi_{o} \cdot\left(1-\left[k \cdot \sin \left(\frac{m \pi}{2 n+1}\right)\right]^{2}\right)^{1 / 2}\right\}\right]
\end{aligned}
$$

The variable $n$ is the number of terms that you want to expand each elliptic integral. Typically, $n=10$ or even less will yield sufficient accuracy. Recall that our goal was to find $\phi_{o}$. The process is as follows: 1) Guess $k$ until (36) is true, 2) Solve (34) for $\phi_{o}$.

\subsection{Horizontal Deflection at the Pole Tip}

After finding $\phi_{o}$ we can determine the horizontal deflection $\delta_{x}$ at the pole tip. To find $\delta_{x}$, we substitute (14) into (5), solve for $\mathrm{dx}$, and then integrate both sides from $\phi=$ to $\phi=\phi_{o}$ to obtain:

$$
\delta_{x}=\sqrt{\frac{E I}{2}} \cdot \int_{0}^{\phi_{o}} \frac{\sin \phi \cdot d \phi}{\sqrt{P_{R} \cdot \sin \left(\phi_{o}+\beta\right)-P_{R} \cdot \sin (\phi+\beta)}}
$$

As it stands, $(39)$ is not integrable and only numerical methods can be used. However, elliptic integrals will once again provide us with a simplified alternative. We define a value $\alpha_{x}$ as:

$$
\alpha_{x}=\frac{P_{R} \cdot \delta_{x}^{2}}{E I}
$$

Substituting (40) into (39) will yield:

$$
\sqrt{\alpha_{x}}=\int_{0}^{\phi_{o}} \frac{1}{\sqrt{2}} \cdot \frac{\sin \phi \cdot d \phi}{\sqrt{\sin \left(\phi_{o}+\beta\right)-\sin (\phi+\beta)}}
$$


By using the same substitutions as in (23) and (29), we can arrive at an equation that is analogous to $(33)$ :

$$
\sqrt{\alpha_{x}}=\int_{\psi_{o}}^{\frac{\pi}{2}} \frac{\sin \phi \cdot d \psi}{\sqrt{1-k^{2} \cdot \sin ^{2}}}
$$

It is obvious that we must find a way to represent the $\sin \phi$ term in the numerator as some function of $\psi$. To this end, we can take $(29)$ and solve for $\phi$ :

$$
\phi=\arcsin \left(2 k^{2} \cdot \sin ^{2} \psi-1\right)-\beta
$$

If we take the sine of both sides of 43 and expand, we get:

$$
\sin \phi=\left(2 k^{2} \cdot \sin ^{2} \psi-1\right) \cdot \cos \beta-\sin \beta \cdot 2 k \cdot \sin \psi \cdot \sqrt{1-k^{2} \cdot \sin ^{2}}
$$

Substitute 44) into 42):

$$
\begin{aligned}
\sqrt{\alpha_{x}}= & \cos \beta \cdot \int_{\psi_{o}}^{\frac{\pi}{2}} \frac{2 k^{2} \sin ^{2} \psi-1}{\sqrt{1-k^{2} \sin ^{2}}} \cdot d \psi-2 k \sin \beta \cdot \int_{\psi_{o}}^{\frac{\pi}{2}} \sin \psi d \psi \\
= & \cos \beta \cdot \int_{\psi_{o}}^{\frac{\pi}{2}} \frac{-2\left(1-k^{2} \sin ^{2} \psi\right)+1}{\sqrt{1-k^{2} \sin ^{2}}} \cdot d \psi+\left.2 k \sin \beta \cdot \cos \psi\right|_{\psi_{o}} ^{\pi / 2} \\
= & -2 \cos \beta \cdot \int_{\psi_{o}}^{\frac{\pi}{2}} \sqrt{1-k^{2} \sin ^{2} \psi} \cdot d \psi+\cos \beta \cdot \int_{\psi_{o}}^{\frac{\pi}{2}} \frac{1}{\sqrt{1-k^{2} \sin ^{2}}} \cdot d \psi \\
& +2 k \sin \beta \cdot\left(0-\cos \psi_{o}\right)
\end{aligned}
$$

As we did with (35), we can rewrite the first term of (45) as the difference of the Complete and Incomplete Elliptic Integrals of the Second Kind, and the second term as the difference of the Complete and Incomplete Elliptic Integral of the First Kind:

$$
\begin{aligned}
\sqrt{\alpha_{x}}= & -2 \cos \beta \cdot\left[E_{n}\left(\frac{\pi}{2}, k\right)-E_{n}\left(\psi_{o}, k\right)\right]+\cos \beta \cdot\left[F_{n}\left(\frac{\pi}{2}, k, 0\right)-F_{n}\left(\psi_{o}, k, 0\right)\right] \\
& -2 k \sin \beta \cos \psi_{o}
\end{aligned}
$$

where:

$$
\begin{aligned}
E_{n}\left(\frac{\pi}{2}, k\right) & =\frac{\pi}{2} \cdot\left[(2 n+1)-2 \cdot(2 n+1)^{-1} \cdot \sum_{m=1}^{n} \frac{\tan ^{2} \theta_{m}}{\rho_{m}}\right] \\
E_{n}\left(\psi_{o}, k\right) & =(2 n+1) \cdot \psi_{o}-2 \cdot(2 n+1)^{-1} \cdot \sum_{m=1}^{n} \frac{\tan ^{2} \theta_{m} \cdot \arctan \left(\rho_{m} \cdot \tan \psi_{o}\right)}{\rho_{m}} \\
\theta_{m} & =\frac{m \pi}{2 n+1} \\
\rho_{m} & =\sqrt{1-k^{2} \cdot \cos ^{2} \theta_{m}}
\end{aligned}
$$

From our prior efforts in solving for $\phi_{o}$ we would have already found $\beta, k$, and $\psi_{o}$, as well as the difference of the Complete and Incomplete Elliptic Integrals of the First Kind. After solving (46) for $\alpha_{x}$, we can substitute this value into 40 to find $\delta_{x}$. 


\subsection{Vertically Deflected Height at the Pole Tip}

Similarly, to find $L-\delta_{y}$ (and $\delta_{y}$ ), substitute (14) into (6), solve for dy, and then integrate both sides from $\phi=$ to $\phi=\phi_{o}$ to obtain:

$$
L-\delta_{y}=\sqrt{\frac{E I}{2}} \cdot \int_{0}^{\phi_{o}} \frac{\cos \phi \cdot d \phi}{\sqrt{P_{R} \cdot \sin \left(\phi_{o}+\beta\right)-P_{R} \cdot \sin (\phi+\beta)}}
$$

As it stands, (51) is not integrable. However, elliptic integrals can be used to obtain a simplified alternative. We define a value $\alpha_{y}$ as:

$$
\alpha_{y}=\frac{P_{R} \cdot\left(L-\delta_{y}\right)^{2}}{E I}
$$

Substituting (52) into (51) will yield:

$$
\sqrt{\alpha_{y}}=\int_{0}^{\phi_{o}} \frac{1}{\sqrt{2}} \cdot \frac{\cos \phi \cdot d \phi}{\sqrt{\sin \left(\phi_{o}+\beta\right)-\sin (\phi+\beta)}}
$$

By using the same substitutions as in (23) and (29), we can arrive at an equation that is analogous to 33 :

$$
\sqrt{\alpha_{y}}=\int_{\psi_{o}}^{\frac{\pi}{2}} \frac{\cos \phi \cdot d \psi}{\sqrt{1-k^{2} \cdot \sin ^{2}}}
$$

It is obvious that we must find a way to represent the $\cos \phi$ term in the numerator as some function of $\psi$. To this end, we can take the cosine of both sides of (43), expand, and obtain:

$$
\cos \phi=\left(2 k^{2} \cdot \sin ^{2} \psi-1\right) \cdot \sin \beta+2 k \cdot \sin \psi \cdot \sqrt{1-k^{2} \sin ^{2} \psi} \cdot \cos \beta
$$

Substitute (55) into (54):

$$
\begin{aligned}
\sqrt{\alpha_{y}}= & \sin \beta \cdot \int_{\psi_{o}}^{\frac{\pi}{2}} \frac{2 k^{2} \sin ^{2} \psi-1}{\sqrt{1-k^{2} \sin ^{2}}} \cdot d \psi+2 k \cos \beta \cdot \int_{\psi_{o}}^{\frac{\pi}{2}} \sin \psi d \psi \\
= & \sin \beta \cdot \int_{\psi_{o}}^{\frac{\pi}{2}} \frac{-2\left(1-k^{2} \sin ^{2} \psi\right)+1}{\sqrt{1-k^{2} \sin ^{2}}} \cdot d \psi-\left.2 k \cos \beta \cdot \cos \psi\right|_{\psi_{o}} ^{\pi / 2} \\
= & -2 \sin \beta \cdot \int_{\psi_{o}}^{\frac{\pi}{2}} \sqrt{1-k^{2} \sin ^{2} \psi} \cdot d \psi+\sin \beta \cdot \int_{\psi_{o}}^{\frac{\pi}{2}} \frac{1}{\sqrt{1-k^{2} \sin ^{2}}} \cdot d \psi \\
& -2 k \cos \beta \cdot\left(0-\cos \psi_{o}\right)
\end{aligned}
$$

As we did with (35), we can rewrite the first term of (56) as the difference of the Complete and Incomplete Elliptic Integrals of the Second Kind, and the second term as the difference of the Complete and Incomplete Elliptic Integral of the First Kind:

$$
\begin{aligned}
\sqrt{\alpha_{y}}= & -2 \sin \beta \cdot\left[E_{n}\left(\frac{\pi}{2}, k\right)-E_{n}\left(\psi_{o}, k\right)\right]+\sin \beta \cdot\left[F_{n}\left(\frac{\pi}{2}, k, 0\right)-F_{n}\left(\psi_{o}, k, 0\right)\right] \\
& +2 k \cos \beta \cos \psi_{o}
\end{aligned}
$$

where every variable and term is already known from our previous efforts at finding $\psi_{o}$ and the horizontal deflection. After solving (57) for $\alpha_{y}$, we can substitute this value into (52) to find $L-\delta_{y}$, from which we can also obtain the vertical deflection $\delta_{y}$. 


\section{Large Deformation using Two Prismatic Elements}

We now consider a pole with point loads at two locations, as shown on the right side of Figure 3 at some Point 1, a horizontal force $P_{1, T}$ and a vertical force $P_{1, V}$ is applied; at some Point 2, a horizontal force $P_{2, T}$ and a vertical force $P_{2, V}$ is applied. The pole is treated as two prismatic elements: the length $L_{1}$ between the base and Point 1 is considered the first element ("Segment 1"); the length $L_{2}$ between Point 1 and Point 2 is considered the second element ("Segment 2"). At Point 1, we will determine the angle of deflection $\phi_{1}$, the horizontal deflection $\delta_{x 1}$, and the vertically deflected height $a$; at Point 2, we will determine the angle of deflection $\phi_{2}$, the horizontal deflection $\delta_{x 2}$ (which is with deflection with respect to Point 1), and the vertically deflected height $b$ (which results from the downward deflection of the second element only and is independent of any bending in the first element).

\subsection{Angles of Deflection at the Element Tips}

We first focus on Segment 2, which appears as a pole with one load. Therefore, we can apply (26) and (29) to Segment 2 as follows:

$$
\begin{gathered}
\sin ^{2} \psi=\frac{1+\sin \left(\phi+\beta_{2}\right)}{1+\sin \left(\phi_{2}+\beta_{2}\right)} \\
k_{2}^{2} \cdot \sin ^{2} \psi=\frac{1+\sin \left(\phi+\beta_{2}\right)}{2}
\end{gathered}
$$

where, like 16$)$, we can express $\beta_{2}$ as:

$$
\beta_{2}=\arcsin \left(\frac{-P_{2, V}}{\sqrt{P_{2, T}^{2}+P_{2, V}^{2}}}\right)
$$

By substituting (59) into (58) and solving, we get:

$$
\sin \left(\phi_{2}+\beta_{2}\right)=2 \cdot k_{2}^{2}-1
$$

Finally:

$$
L_{2}=\sqrt{\frac{E I_{2}}{P_{2, R}}} \cdot\left[F_{n}\left(\frac{\pi}{2}, k_{2}, 0\right)-F_{n}\left(\left.\psi_{2}\right|_{\phi=\phi_{1}}, k_{2}, 0\right)\right]
$$

where

$$
P_{2, R}=\sqrt{P_{2, T}^{2}+P_{2, V}^{2}}
$$

and

$$
\left.\psi_{2}\right|_{\phi=\phi_{1}}=\arcsin \left(\frac{1}{k_{2}} \cdot \sqrt{\frac{1+\sin \left(\phi_{1}+\beta_{2}\right)}{2}}\right)
$$

The moment anywhere along Segment 1 can be computed:

$$
M(s)=P_{2, T} \cdot(a+b-y)+P_{1, T} \cdot(a-y)+P_{2, V} \cdot\left(\delta_{x 1}+\delta_{x 2}-x\right)+P_{1, V} \cdot\left(\delta_{x 1}-x\right)
$$


Differentiate both sides of 65 with respect to $s$ to obtain:

$$
\begin{aligned}
\frac{d M}{d s} & =-\left(P_{2, T}+P_{1, T}\right) \cdot \frac{d y}{d s}-\left(P_{2, V}+P_{1, V}\right) \cdot \frac{d x}{d s} \\
& =-\left(P_{2, T}+P_{1, T}\right) \cdot \cos \phi-\left(P_{2, V}+P_{1, V}\right) \cdot \sin \phi
\end{aligned}
$$

Substitute (66) into (2) to get:

$$
E I_{1} \cdot \frac{d^{2} \phi}{d s^{2}}+\left(P_{2, T}+P_{1, T}\right) \cdot \cos \phi+\left(P_{2, V}+P_{1, V}\right) \cdot \sin \phi=0
$$

After multiplying both sides of (67) by $d \phi / d s$, we can rewrite the equation as follows:

$$
\frac{d}{d s}\left[\frac{1}{2} \cdot E I_{1} \cdot\left(\frac{d \phi}{d s}\right)^{2}+\left(P_{2, T}+P_{1, T}\right) \cdot \sin \phi-\left(P_{2, V}+P_{1, V}\right) \cdot \cos \phi\right]=0
$$

From here, we can define the differentiation constant $C$ as:

$$
C=\frac{1}{2} \cdot E I_{1} \cdot\left(\frac{d \phi}{d s}\right)^{2}+\left(P_{2, T}+P_{1, T}\right) \cdot \sin \phi-\left(P_{2, V}+P_{1, V}\right) \cdot \cos \phi
$$

To solve for $C$, we consider (14), and derive a similar equation:

$$
\frac{d \phi}{d s}=\sqrt{\frac{2}{E I_{2}}} \cdot \sqrt{P_{2, R} \cdot \sin \left(\phi_{2}+\beta_{2}\right)-P_{2, R} \cdot \sin \left(\phi+\beta_{2}\right)} \text { for } \phi_{1} \leq \phi \leq \phi_{2}
$$

Substitute $\phi=\phi_{1}$ and $d \phi / d s=d \phi /\left.d s\right|_{\phi=\phi_{1}}$ into 69 and solving, we find that the constant $C$ is:

$$
C=P_{2, R} \cdot \sin \left(\phi_{2}+\beta_{2}\right)+P_{1, R} \cdot \sin \left(\phi_{1}+\beta_{1}\right)
$$

where $P_{1, R}$ and $\beta_{1}$ is given by:

$$
\begin{aligned}
P_{1, R} & =\sqrt{P_{1, T}^{2}+P_{1, V}^{2}} \\
\beta_{1} & =\arcsin \left(\frac{-P_{1, V}}{\sqrt{P_{1, T}^{2}+P_{1, V}^{2}}}\right)
\end{aligned}
$$

Substitute the value for $C$ back into the equation and solving, we obtain:

$$
\frac{d \phi}{d s}=\sqrt{\frac{2}{E I_{1}}} \cdot \sqrt{P_{2, R} \cdot \sin \left(\phi_{2}+\beta_{2}\right)+P_{1, R} \cdot \sin \left(\phi_{1}+\beta_{1}\right)-P_{2, R} \cdot \sin \left(\phi+\beta_{2}\right)-P_{1, R} \cdot \sin \left(\phi+\beta_{1}\right)}
$$

Using trigonometric manipulation, the latter two terms can be rewritten as:

$$
P_{2, R} \cdot \sin \left(\phi+\beta_{2}\right)+P_{1, R} \cdot \sin \left(\phi+\beta_{1}\right)=A \cdot \sin \left(\phi-\frac{\pi}{2}-\theta\right)
$$


where

$$
\begin{aligned}
A & =\sqrt{P_{2, R}^{2}+P_{1, R}^{2}+2 \cdot P_{2, R} \cdot P_{1, R} \cdot \cos \left(\beta_{2}-\beta_{1}\right)} \\
\theta & =\arctan \left(\frac{P_{2, R} \cdot \cos \beta_{2}+P_{1, R} \cdot \cos \beta_{1}}{P_{2, R} \cdot \sin \beta_{2}+P_{1, R} \cdot \sin \beta_{1}}\right)
\end{aligned}
$$

We can now manipulate $(74)$ to include $A$ :

$$
\frac{d \phi}{d s}=\sqrt{\frac{2 \cdot A}{E I_{1}}} \cdot \sqrt{\frac{P_{2, R}}{A} \cdot \sin \left(\phi_{2}+\beta_{2}\right)+\frac{P_{1, R}}{A} \cdot \sin \left(\phi_{1}+\beta_{1}\right)-\sin \left(\phi-\frac{\pi}{2}-\theta\right)}
$$

Now we find $L_{1}$ by solving $(78)$ for $d s$ and then integrating $d s$ from 0 to $\phi_{1}$ :

$$
\begin{aligned}
L_{1} & =\int_{0}^{\phi_{1}} d s \\
& =\sqrt{\frac{E I_{1}}{2 \cdot A}} \cdot \int_{0}^{\phi_{1}} \frac{d \phi}{\sqrt{\frac{P_{2, R}}{A} \cdot \sin \left(\phi_{2}+\beta_{2}\right)+\frac{P_{1, R}}{A} \cdot \sin \left(\phi_{1}+\beta_{1}\right)-\sin \left(\phi-\frac{\pi}{2}-\theta\right)}}
\end{aligned}
$$

So we let:

$$
\sin ^{2} \psi=\frac{1+\sin \left(\phi-\frac{\pi}{2}-\theta\right)}{1+\frac{P_{2, R}}{A} \cdot \sin \left(\phi_{2}+\beta_{2}\right)+\frac{P_{1, R}}{A} \cdot \sin \left(\phi_{1}+\beta_{1}\right)}
$$

Solving 80 , we get:

$$
\frac{P_{2, R}}{A} \cdot \sin \left(\phi_{2}+\beta_{2}\right)+\frac{P_{1, R}}{A} \cdot \sin \left(\phi_{1}+\beta_{1}\right)=\frac{1+\sin \left(\phi-\frac{\pi}{2}-\theta\right)}{\sin ^{2}}-1
$$

Substituting into (81) into 79 and rearranging, we get:

$$
L_{1}=\sqrt{\frac{E I_{1}}{2 \cdot A}} \cdot \int_{0}^{\phi_{1}} \frac{\sin \psi \cdot d \phi}{\sqrt{\left[1+\sin \left(\phi-\frac{\pi}{2}-\theta\right)\right] \cdot\left(1-\sin ^{2}\right)}}
$$

Let:

$$
k_{1}^{2} \cdot \sin ^{2}=\frac{1+\sin \left(\phi-\frac{\pi}{2}-\theta\right)}{2}
$$

Solving 83 gives us:

$$
\sin \left(\phi-\frac{\pi}{2}-\theta\right)=2 \cdot k_{1}^{2} \cdot \sin ^{2} \psi-1
$$

Substituting (84) into 81 gives:

$$
\frac{P_{2, R}}{A} \cdot \sin \left(\phi_{2}+\beta_{2}\right)+\frac{P_{1, R}}{A} \cdot \sin \left(\phi_{1}+\beta_{1}\right)=2 \cdot k_{1}{ }^{2}-1
$$


Substituting 61 into 85 gives a governing equation:

$$
\frac{P_{2, R}}{A} \cdot\left(2 k_{2}^{2}-1\right)+\frac{P_{1, R}}{A} \cdot \sin \left(\phi_{1}+\beta_{1}\right)=2 \cdot k_{1}^{2}-1
$$

Additionally, by applying the Pythagorean Theorem to (84), we get:

$$
\cos \left(\phi-\frac{\pi}{2}-\theta\right)=2 \cdot k_{1} \cdot \sin \psi \cdot \sqrt{1-k_{1}^{2} \cdot \sin ^{2}}
$$

Differentiating both sides of 87 gives:

$$
\begin{aligned}
\cos \left(\phi-\frac{\pi}{2}-\theta\right) \cdot d \phi & =2 \cdot k_{1}{ }^{2} \cdot 2 \cdot \sin \psi \cdot \cos \psi \cdot d \psi \\
d \phi & =\frac{2 \cdot k_{1} \cdot \cos \psi \cdot d \psi}{\sqrt{1-k_{1}^{2} \cdot \sin ^{2}}}
\end{aligned}
$$

Substituting into the equation for $L_{1}$, we get:

$$
L_{1}=\sqrt{\frac{E I_{1}}{A}} \cdot \int_{0}^{\phi_{1}} \frac{d \psi}{\sqrt{1-k_{1}^{2} \cdot \sin ^{2}}}
$$

Now we need to change the limits of integration. Substituting $\phi=\phi_{1}$ into (84) and solving for $\psi$, the upper limit of integration becomes:

$$
\left.\psi_{1}\right|_{\phi=\phi_{1}}=\arcsin \left[\frac{1}{\sqrt{2} \cdot k_{1}} \cdot \sqrt{1+\sin \left(\phi_{1}-\frac{\pi}{2}-\theta\right)}\right]
$$

Substituting $\phi=0$ into (84) and solving for $\psi$, the lower limit of integration becomes:

$$
\left.\psi_{1}\right|_{\phi=0}=\arcsin \left[\frac{1}{\sqrt{2} \cdot k_{1}} \cdot \sqrt{1+\sin \left(-\frac{\pi}{2}-\theta\right)}\right]
$$

So, we can rewrite 89 as:

$$
\begin{aligned}
L_{1} & =\sqrt{\frac{E I_{1}}{A}} \cdot\left[\int_{0}^{\psi_{1}\left(\phi=\phi_{1}\right)} \frac{d \psi}{\sqrt{1-k_{1}^{2} \cdot \sin ^{2}}}-\int_{0}^{\psi_{1}(\phi=0)} \frac{d \psi}{\sqrt{1-k_{1}^{2} \cdot \sin ^{2}}}\right] \\
& =\sqrt{\frac{E I_{1}}{A}} \cdot\left[F_{n}\left(\psi_{1}\left(\phi=\phi_{1}\right), k_{1}, 0\right)-F_{n}\left(\psi_{1}(\phi=0), k_{1}, 0\right)\right]
\end{aligned}
$$

To solve for the tip angles $\phi_{1}$ and $\phi_{2}$, we follow this process: 1) Guess a value for $\phi_{1}, 2$ ) Adjust $k_{1}$ until (92) is true, 3) Adjust $k_{2}$ until (62) is true, 4) Verify to see if (86) holds true. If (86) does not hold true, then a new guess for $\phi_{1}$ will be required. 


\subsection{Solving for $\delta_{x 1}$, the Horizontal Deflection of Segment 1}

We know $\mathrm{dx} / \mathrm{ds}=\sin \phi$ and $(79)$. Solve for $\mathrm{ds}$, substitute into $\mathrm{dx} / \mathrm{ds}$, and solve for $\mathrm{dx}$ :

$$
\begin{aligned}
d x & =d s \cdot \sin \phi \\
& =\frac{\sin \phi \cdot d \phi}{\sqrt{\frac{2 A}{E I_{1}}} \cdot \sqrt{\frac{P_{2, R}}{A} \cdot \sin \left(\phi_{2}+\beta_{2}\right)+\frac{P_{1, R}}{A} \cdot \sin \left(\phi_{1}+\beta_{1}\right)-\sin \left(\phi-\frac{\pi}{2}-\theta\right)}}
\end{aligned}
$$

Now integrating from $\phi=0$ to $\phi=\phi_{1}$ to get:

$$
\delta_{x 1}=\sqrt{\frac{E I_{1}}{2 A}} \cdot \int_{0}^{\phi_{1}} \frac{\sin \phi \cdot d \phi}{\sqrt{\frac{P_{2, R}}{A} \cdot \sin \left(\phi_{2}+\beta_{2}\right)+\frac{P_{1, R}}{A} \cdot \sin \left(\phi_{1}+\beta_{1}\right)-\sin \left(\phi-\frac{\pi}{2}-\theta\right)}}
$$

Perform the same substitutions as before to get into the elliptic integral format:

$$
\delta_{x 1}=\sqrt{\frac{E I_{1}}{A}} \cdot \int_{\psi_{1}(\phi=0)}^{\psi_{1}\left(\phi=\phi_{1}\right)} \frac{\sin \phi \cdot d \psi}{\sqrt{1-k_{1}^{2} \cdot \sin ^{2}}}
$$

To express $\sin \phi$ in terms of $\psi$, we take (84) and solve for $\phi$ :

$$
\begin{aligned}
\sin \left(\phi-\frac{\pi}{2}-\theta\right) & =2 \cdot k_{1}^{2} \cdot \sin ^{2}-1 \\
\phi-\frac{\pi}{2}-\theta & =\arcsin \left(2 \cdot k_{1}{ }^{2} \cdot \sin ^{2}-1\right) \\
\phi & =\arcsin \left(2 \cdot{k_{1}}^{2} \cdot \sin ^{2}-1\right)+\frac{\pi}{2}+\theta
\end{aligned}
$$

Taking the sine of both sides will yield:

$$
\begin{aligned}
\sin \phi & =\sin \left[\arcsin \left(2 \cdot k_{1}{ }^{2} \cdot \sin ^{2}-1\right)+\left(\frac{\pi}{2}+\theta\right)\right] \\
& =\left(2 \cdot k_{1}{ }^{2} \cdot \sin ^{2}-1\right) \cdot \cos \left(\frac{\pi}{2}+\theta\right)+\cos \left[\arcsin \left(2 \cdot k_{1}{ }^{2} \cdot \sin ^{2}-1\right)\right] \cdot \sin \left(\frac{\pi}{2}+\theta\right) \\
& =\left(2 \cdot k_{1}{ }^{2} \cdot \sin ^{2}-1\right) \cdot \cos \left(\frac{\pi}{2}+\theta\right)+2 \cdot k_{1} \cdot \sin \psi \cdot \sqrt{1-k_{1}{ }^{2} \cdot \sin ^{2} \psi} \cdot \sin \left(\frac{\pi}{2}+\theta\right)
\end{aligned}
$$


Now, substituting back into our equation for $\delta_{x 1}$ we get:

$$
\begin{aligned}
& \delta_{x 1}=\sqrt{\frac{E I_{1}}{A}} \cdot \int_{\psi_{1}(\phi=0)}^{\psi_{1}\left(\phi=\phi_{1}\right)} \frac{\left(2 \cdot k_{1}^{2} \cdot \sin ^{2}-1\right) \cdot \cos \left(\frac{\pi}{2}+\theta\right) \cdot d \psi}{\sqrt{1-k_{1}^{2} \cdot \sin ^{2}}} \\
& +\sqrt{\frac{E I_{1}}{A}} \cdot \int_{\psi_{1}(\phi=0)}^{\psi_{1}\left(\phi=\phi_{1}\right)} 2 \cdot k_{1} \cdot \sin \psi \cdot \sin \left(\frac{\pi}{2}+\theta\right) \cdot d \psi \\
& =\sqrt{\frac{E I_{1}}{A}} \cdot \cos \left(\frac{\pi}{2}+\theta\right) \cdot \int_{\psi_{1}(\phi=0)}^{\psi_{1}\left(\phi=\phi_{1}\right)} \frac{-2 \cdot\left(1-k_{1}{ }^{2} \cdot \sin ^{2}\right)+1}{\sqrt{1-k_{1}^{2} \cdot \sin ^{2}}} \cdot d \psi \\
& -\left.\sqrt{\frac{E I_{1}}{A}} \cdot \sin \left(\frac{\pi}{2}+\theta\right) \cdot 2 \cdot k_{1} \cdot \cos \psi\right|_{\psi_{1}(\phi=0)} ^{\psi_{1}\left(\phi=\phi_{1}\right)} \\
& =-2 \cdot \sqrt{\frac{E I_{1}}{A}} \cdot \cos \left(\frac{\pi}{2}+\theta\right) \cdot \int_{\psi_{1}(\phi=0)}^{\psi_{1}\left(\phi=\phi_{1}\right)} \sqrt{1-k_{1}^{2} \cdot \sin ^{2} \psi} \cdot d \psi \\
& +\sqrt{\frac{E I_{1}}{A}} \cdot \cos \left(\frac{\pi}{2}+\theta\right) \cdot \int_{\psi_{1}(\phi=0)}^{\psi_{1}\left(\phi=\phi_{1}\right)} \frac{d \psi}{\sqrt{1-k_{1}^{2} \cdot \sin ^{2}}} \\
& -\sqrt{\frac{E I_{1}}{A}} \cdot \sin \left(\frac{\pi}{2}+\theta\right) \cdot 2 \cdot k_{1} \cdot\left[\cos \left(\psi_{1}\left(\phi=\phi_{1}\right)\right)-\cos \left(\psi_{1}(\phi=0)\right)\right] \\
& =-2 \cdot \sqrt{\frac{E I_{1}}{A}} \cdot \cos \left(\frac{\pi}{2}+\theta\right) \cdot\left[E_{n}\left(\psi_{1}\left(\phi=\phi_{1}\right), k_{1}\right)-E_{n}\left(\psi_{1}(\phi=0), k_{1}\right)\right] \\
& +\sqrt{\frac{E I_{1}}{A}} \cdot \cos \left(\frac{\pi}{2}+\theta\right) \cdot\left[F_{n}\left(\psi_{1}\left(\phi=\phi_{1}\right), k_{1}, 0\right)-F_{n}\left(\psi_{1}(\phi=0), k_{1}, 0\right)\right] \\
& -\sqrt{\frac{E I_{1}}{A}} \cdot \sin \left(\frac{\pi}{2}+\theta\right) \cdot 2 \cdot k_{1} \cdot\left[\cos \left(\psi_{1}\left(\phi=\phi_{1}\right)\right)-\cos \left(\psi_{1}(\phi=0)\right)\right]
\end{aligned}
$$

\subsection{Solving for a, the Vertically Deflected Height of Segment 1}

We know $\mathrm{dy} / \mathrm{ds}=\cos \phi$ and (79). Solving for dy, integrating from $\phi=0$ to $\phi=\phi_{1}$, and performing substitutions, we get:

$$
a=\sqrt{\frac{E I_{1}}{A}} \cdot \int_{\psi_{1}(\phi=0)}^{\psi_{1}\left(\phi=\phi_{1}\right)} \frac{\cos \phi \cdot d \psi}{\sqrt{1-k_{1}^{2} \cdot \sin ^{2}}}
$$

Now we express $\cos \phi$ in terms of $\psi$ :

$$
\begin{aligned}
\cos \phi & =\cos \left[\arcsin \left(2 \cdot k_{1}{ }^{2} \cdot \sin ^{2} \psi-1\right)+\left(\frac{\pi}{2}+\theta\right)\right] \\
& =\cos \left[\arcsin \left(2 \cdot k_{1}{ }^{2} \cdot \sin ^{2} \psi-1\right)\right] \cdot \cos \left(\frac{\pi}{2}+\theta\right)-\left(2 \cdot k_{1}{ }^{2} \cdot \sin ^{2}-1\right) \cdot \sin \left(\frac{\pi}{2}+\theta\right) \\
& =2 \cdot k_{1} \cdot \sin \psi \cdot \sqrt{1-k_{1}{ }^{2} \cdot \sin ^{2} \psi} \cdot \cos \left(\frac{\pi}{2}+\theta\right)-\left(2 \cdot k_{1}{ }^{2} \cdot \sin ^{2}-1\right) \cdot \sin \left(\frac{\pi}{2}+\theta\right)
\end{aligned}
$$


Substituting back into the equation for $a$, we get:

$$
\begin{aligned}
& a=\sqrt{\frac{E I_{1}}{A}} \cdot \int_{\psi_{1}(\phi=0)}^{\psi_{1}\left(\phi=\phi_{1}\right)} 2 \cdot k_{1} \cdot \sin \psi \cdot d \psi \cdot \cos \left(\frac{\pi}{2}+\theta\right) \\
& -\sqrt{\frac{E I_{1}}{A}} \cdot \int_{\psi_{1}(\phi=0)}^{\psi_{1}\left(\phi=\phi_{1}\right)} \frac{-2 \cdot\left(1-k_{1}^{2} \cdot \sin ^{2} \psi\right)+1}{\sqrt{1-k_{1}^{2} \cdot \sin ^{2}}} \cdot d \psi \cdot \sin \left(\frac{\pi}{2}+\theta\right) \\
& =\left.\sqrt{\frac{E I_{1}}{A}} \cdot \cos \left(\frac{\pi}{2}+\theta\right) \cdot 2 k_{1} \cdot(-\cos \psi)\right|_{\psi_{1}(\phi=0)} ^{\psi_{1}\left(\phi=\phi_{1}\right)} \\
& -\sqrt{\frac{E I_{1}}{A}} \cdot \sin \left(\frac{\pi}{2}+\theta\right) \cdot(-2) \cdot \int_{\psi_{1}(\phi=0)}^{\psi_{1}\left(\phi=\phi_{1}\right)} \sqrt{1-k_{1}^{2} \cdot \sin ^{2} \psi} \cdot d \psi \\
& -\sqrt{\frac{E I_{1}}{A}} \cdot \sin \left(\frac{\pi}{2}+\theta\right) \cdot \int_{\psi_{1}(\phi=0)}^{\psi_{1}\left(\phi=\phi_{1}\right)} \frac{1}{\sqrt{1-k_{1}^{2} \cdot \sin ^{2}}} \cdot d \psi \\
& =-\sqrt{\frac{E I_{1}}{A}} \cdot \cos \left(\frac{\pi}{2}+\theta\right) \cdot 2 k_{1} \cdot\left[\cos \left(\psi_{1}\left(\phi=\phi_{1}\right)\right)-\cos \left(\psi_{1}(\phi=0)\right)\right] \\
& +2 \cdot \sqrt{\frac{E I_{1}}{A}} \cdot \sin \left(\frac{\pi}{2}+\theta\right) \cdot\left[E_{n}\left(\psi_{1}\left(\phi=\phi_{1}\right), k_{1}\right)-E_{n}\left(\psi_{1}(\phi=0), k_{1}\right)\right] \\
& -\sqrt{\frac{E I_{1}}{A}} \cdot \sin \left(\frac{\pi}{2}+\theta\right) \cdot\left[F_{n}\left(\psi_{1}\left(\phi=\phi_{1}\right), k_{1}, 0\right)-F_{n}\left(\psi_{1}(\phi=0), k_{1}, 0\right)\right]
\end{aligned}
$$

\subsection{Solving for $\delta_{x 2}$, the Horizontal Deflection of Segment 2}

Based on the results of the single load case, we can write:

$$
\begin{aligned}
\delta_{x 2}= & -\sqrt{\frac{E I}{P_{2, R}}} \cdot 2 \cos \beta_{2} \cdot\left[E_{n}\left(\frac{\pi}{2}, k_{2}\right)-E_{n}\left(\psi_{2}\left(\phi=\phi_{1}\right), k_{2}\right)\right] \\
& +\sqrt{\frac{E I}{P_{2, R}}} \cdot \cos \beta_{2} \cdot\left[F_{n}\left(\frac{\pi}{2}, k_{2}, 0\right)-F_{n}\left(\psi_{2}\left(\phi=\phi_{1}\right), k_{2}, 0\right)\right] \\
& -\sqrt{\frac{E I}{P_{2, R}}} \cdot 2 k_{2} \cdot \sin \beta_{2} \cdot \cos \left(\psi_{2}\left(\phi=\phi_{1}\right)\right)
\end{aligned}
$$




\subsection{Solving for $b$, the Vertically Deflected Height of Segment 2}

Based on the results of the single load case, we can write:

$$
\begin{aligned}
b= & -\sqrt{\frac{E I}{P_{2, R}}} \cdot 2 \sin \beta_{2} \cdot\left[E_{n}\left(\frac{\pi}{2}, k_{2}\right)-E_{n}\left(\psi_{2}\left(\phi=\phi_{1}\right), k_{2}\right)\right] \\
& +\sqrt{\frac{E I}{P_{2, R}}} \cdot \sin \beta_{2} \cdot\left[F_{n}\left(\frac{\pi}{2}, k_{2}, 0\right)-F_{n}\left(\psi_{2}\left(\phi=\phi_{1}\right), k_{2}, 0\right)\right] \\
& +\sqrt{\frac{E I}{P_{2, R}}} \cdot 2 k_{2} \cdot \cos \beta_{2} \cdot \cos \left(\psi_{2}\left(\phi=\phi_{1}\right)\right)
\end{aligned}
$$

\section{Validation: Comparing the Analytical Solution to FEA}

As a validation step, we will compare the deflection angles calculated from the Analytical Solution to those calculated from Finite Element Analysis (FEA). We first approach the 1element case shown in Figure 2, the results of which are given in Table 1. We then approach the 2-element case shown in Figure 3, the results of which are given in Table 2. In all cases, the tip angles calculated from the Analytical Solution are nearly identical to the same values calculated using FEA.

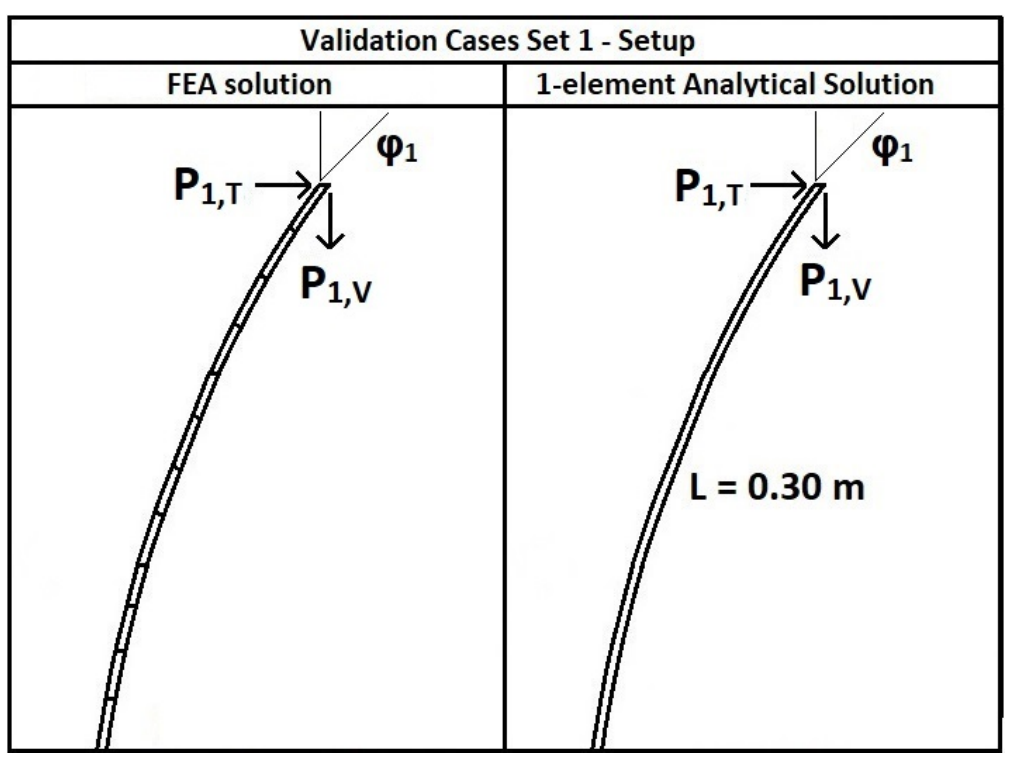

Figure 2: Loading conditions in validation cases set 1. 
Table 1: 1-element Pole ${ }^{\mathrm{a}}$ with a Tip Load ${ }^{\mathrm{b}}$ : Analytical Solution versus FEA

\begin{tabular}{|l|l|l|l|}
\hline Validation Cases Set 1 & FEA & \multicolumn{2}{|l|}{ Analytical } \\
\hline Point Load 1 & $\phi_{1}$ & $\phi_{1}$ & $\mathrm{k}$ \\
\hline$P_{T 1}=3.92, P_{V 1}=0$ & $36.34^{\circ}$ & $36.10^{\circ}$ & 0.89139 \\
\hline$P_{T 1}=3.92, P_{V 1}=0.2$ & $37.15^{\circ}$ & $36.91^{\circ}$ & 0.88291 \\
\hline$P_{T 1}=3.92, P_{V 1}=0.4$ & $38.00^{\circ}$ & $37.76^{\circ}$ & 0.87433 \\
\hline$P_{T 1}=3.92, P_{V 1}=1$ & $40.71^{\circ}$ & $40.49^{\circ}$ & 0.84887 \\
\hline$P_{T 1}=3.92, P_{V 1}=2$ & $45.87^{\circ}$ & $45.71^{\circ}$ & 0.81249 \\
\hline$P_{T 1}=3.92, P_{V 1}=3$ & $51.87^{\circ}$ & $51.80^{\circ}$ & 0.79002 \\
\hline$P_{T 1}=3.92, P_{V 1}=3.92$ & $58.09^{\circ}$ & $58.12^{\circ}$ & 0.78329 \\
\hline$P_{T 1}=3.92, P_{V 1}=6$ & $73.91^{\circ}$ & $74.07^{\circ}$ & 0.80504 \\
\hline$P_{T 1}=3.92, P_{V 1}=8$ & $89.37^{\circ}$ & $89.43^{\circ}$ & 0.84589 \\
\hline$P_{T 1}=3.92, P_{V 1}=9$ & $96.53^{\circ}$ & $96.49^{\circ}$ & 0.86612 \\
\hline
\end{tabular}

${ }^{a}$ The pole has length of $\mathrm{L}=0.3 \mathrm{~m}$ and flexural rigidity $\mathrm{EI}=0.24$.

${ }^{\mathrm{b}}$ All point loads are in Newtons.

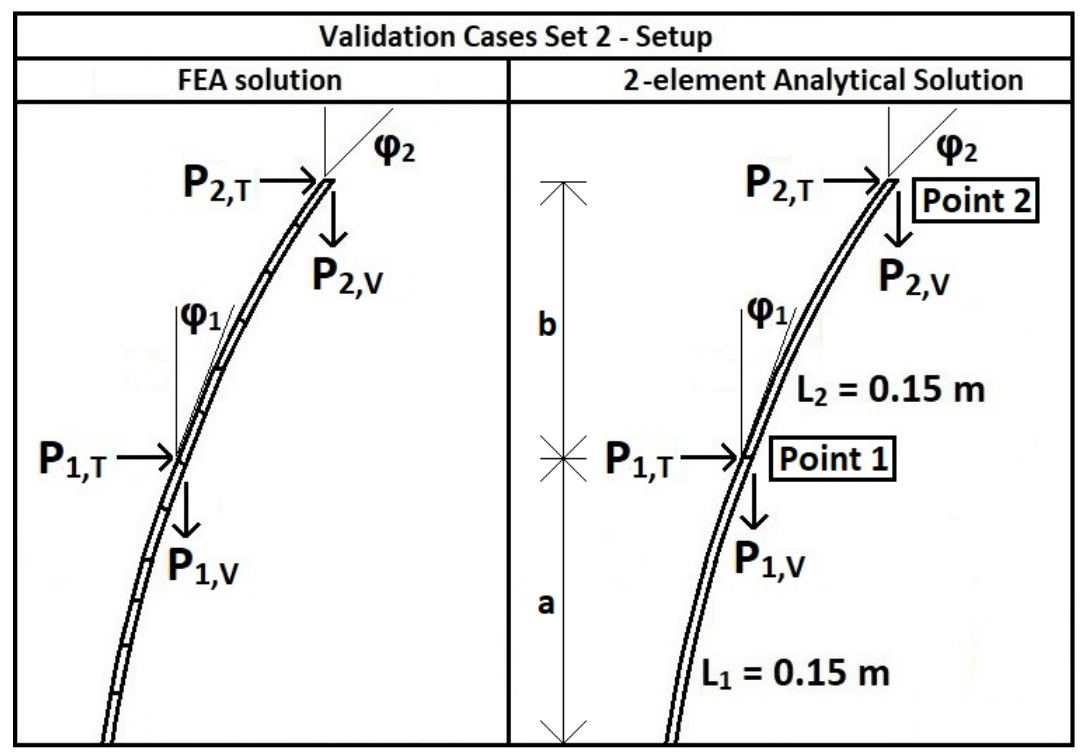

Figure 3: Loading conditions in validation cases set 2. 
Table 2: 2-element Pole ${ }^{\mathrm{a}}$ with 2 Tip Loads ${ }^{\mathrm{b}}$ : Analytical Solution versus FEA

\begin{tabular}{|l|l|l|l|l|l|l|l|}
\hline \multicolumn{2}{|l|}{ Validation Cases Set 2} & FEA & \multicolumn{4}{l|}{ Analytical } \\
\hline Point Load 2 & Point Load 1 & $\phi_{2}$ & $\phi_{1}$ & $\phi_{2}$ & $\phi_{1}$ & $k_{2}$ & $k_{1}$ \\
\hline$F_{T 2}=3.92, F_{V 2}=0$ & $F_{T 1}=3.92, F_{V 1}=0$ & $43.09^{\circ}$ & $35.19^{\circ}$ & $42.80^{\circ}$ & $34.89^{\circ}$ & 0.91628 & 0.90174 \\
\hline$F_{T 2}=3, F_{V 2}=0$ & $F_{T 1}=3, F_{V 1}=0$ & $35.35^{\circ}$ & $28.66^{\circ}$ & $35.11^{\circ}$ & $28.42^{\circ}$ & 0.88742 & 0.87328 \\
\hline$F_{T 2}=2, F_{V 2}=0$ & $F_{T 1}=2, F_{V 1}=0$ & $25.18^{\circ}$ & $20.28^{\circ}$ & $25.13^{\circ}$ & $20.22^{\circ}$ & 0.84394 & 0.83220 \\
\hline$F_{T 2}=1, F_{V 2}=0$ & $F_{T 1}=1, F_{V 1}=0$ & $13.20^{\circ}$ & $10.58^{\circ}$ & $13.20^{\circ}$ & $10.58^{\circ}$ & 0.78370 & 0.77644 \\
\hline$F_{T 2}=2, F_{V 2}=0$ & $F_{T 1}=1, F_{V 1}=0$ & $22.89^{\circ}$ & $17.90^{\circ}$ & $22.80^{\circ}$ & $17.82^{\circ}$ & 0.83294 & 0.82368 \\
\hline$F_{T 2}=2, F_{V 2}=2$ & $F_{T 1}=2, F_{V 1}=2$ & $35.84^{\circ}$ & $28.38^{\circ}$ & $36.24^{\circ}$ & $28.76^{\circ}$ & 0.65106 & 0.62624 \\
\hline$F_{T 2}=4, F_{V 2}=2$ & $F_{T 1}=2, F_{V 1}=2$ & $52.36^{\circ}$ & $41.33^{\circ}$ & $52.29^{\circ}$ & $41.31^{\circ}$ & 0.84676 & 0.78858 \\
\hline$F_{T 2}=5, F_{V 2}=1$ & $F_{T 1}=2, F_{V 1}=2$ & $52.61^{\circ}$ & $41.95^{\circ}$ & $52.36^{\circ}$ & $41.77^{\circ}$ & 0.91016 & 0.84248 \\
\hline$F_{T 2}=1, F_{V 2}=5$ & $F_{T 1}=2, F_{V 1}=2$ & $48.88^{\circ}$ & $37.34^{\circ}$ & $50.96^{\circ}$ & $39.08^{\circ}$ & 0.51706 & 0.56990 \\
\hline
\end{tabular}

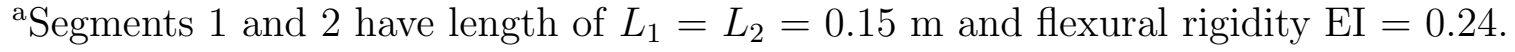

${ }^{\mathrm{b}}$ All point loads are in Newtons.

\section{Conclusion}

The research has presented a methodology for analyzing the large deformation of a cantilever, e.g. a pole, with point loads at two points along the cantilever (and where each point load is allowed to have both a horizontal and a vertical component of force). The research is novel because the analytical solution for more than one tip load along a cantilever has not been published. It is hoped that the methodology contained herein will be of benefit to the field of non-linear mechanics. 


\section{References}

[1] TARSICIO BELENDEZ, CRISTIAN NEIPP, AUGUSTO BELENDEZ, Large and small deflections of a cantilever beam, Eur. J. Phys., 23 (2002), pp. 371-379.

[2] ZAHID SIDDIQI, A study of power transmission poles, Asian J. Civ. Eng., 6 (2005), pp. $511-532$.

[3] RAJENDRA ROKADE, BALAGOPAL R., PRASAD NAPA, Simplified model to predict deflection and natural frequency of steel pole structures, J. Inst. Eng. India Ser. A, 99 (2018), pp. 595-607.

[4] PANDIT D., BHAKTI PATEL, N. THOMAS, S.M. SRINIVASAN, Finite deflection of slender cantilever with predefined load application locus using an incremental formulation, Comput. Mater. Contin., 45 (2015), pp. 127-144.

[5] IBRAHIM ABU-ALSHAIKH, HASHEM S. ALKHALDI, NABIL BEITHOU, Large deflection of prismatic cantilever beam exposed to combination of end inclined force and tip moment, Mod. Appl. Sci., 12 (2018), pp. 98-111.

[6] T. KOCATURK, S.D. AKBAS, M. SIMSEK, Large deflection static analysis of a cantilever beam subjected to a point load, IJEAS, 2 (2010), pp. 1-13.

[7] HAI-JUN SU, A pseudo-rigid-body 3R model for determining large deflection of cantilever beams subject to tip loads, J Mech. Robot, 1 (2009), pp. 1-11.

[8] AMIN KIMIAEIFAR, JUST HERDER, AMIN BARARI, Large deflection analysis of cantilever beam under end point and distributed loads, J. Chin. Inst. Eng., 37 (2014), pp. 438-445.

[9] LI CHEN, An integral approach for large deflection cantilever beams, Int. J. Nonlin. Mech., 45 (2010), pp. 301-305.

[10] BRYCE L. BARTON, MAHESH S. SHETTY, VICTOR BIRMAN, LOKESWARAPPA R. DHARANI, Tapered cylindrical cantilever beam retrofitted with steel reinforced polymer or grout, Compos. Part B-Eng., 42 (2011), pp. 207-216.

[11] KYUNGWOO LEE, Large deflection of viscoelastic fiber beams, Text. Res. J., 77 (2007), pp. 47-51.

[12] MIHA BROJAN, MATJAZ CEBRON, FRANC KOSEL, Large deflections of nonprismatic nonlinearly elastic cantilever beams subjected to non-uniform continuous load and a concentrated load at the free end, CSTAM, 28 (2012), pp. 863-869.

[13] JOHN C. VISNER, Analytical and experimental analysis of the large deflection of a cantilever beam subjected to a constant, concentrated force, with a constant angle, applied at the free end, Master's thesis, The University of Akron, 2007. 
[14] K.E. BISSHOPP, D.C. DRUCKER, Large deflection of cantilever beams, Quart. Appl. Math., 3 (1945), pp. 272-275.

[15] R. FRISCH-FAY, Flexible Bars, Buttersworth, London, 1962. 